\title{
Intact SMAD-4 is a predictor of increased locoregional recurrence in upfront resected pancreas cancer receiving adjuvant therapy
}

\author{
Hunter C. Gits ${ }^{1}$, Amy H. Tang', William S. Harmsen ${ }^{3}$, William R. Bamlet ${ }^{3}$, Rondell P. Graham ${ }^{4}$, \\ Gloria M. Petersen ${ }^{5}$, Thomas C. Smyrk ${ }^{4}$, Amit Mahipal ${ }^{6}$, Roman O. Kowalchuk ${ }^{1}$, Jonathan B. Ashman ${ }^{7}$, \\ William G. Rule ${ }^{7}$, Dawn Owen ${ }^{1}$, Michelle A. Neben Wittich ${ }^{1}$, Robert R. McWilliams ${ }^{6}$, \\ Thorvardur Halfdanarson ${ }^{6}$, Wen Wee $\mathrm{Ma}^{6}$, Terence T. Sio ${ }^{7}$, Sean P. Cleary ${ }^{8}$, Mark J. Truty ${ }^{8}$, \\ Michael G. Haddock ${ }^{1}$, Christopher L. Hallemeier ${ }^{1}$, Kenneth W. Merrell ${ }^{1}$
}

${ }^{1}$ Department of Radiation Oncology, Mayo Clinic, Rochester, MN, USA; ${ }^{2}$ Leroy T. Canoles Jr. Cancer Research Center, Department of Microbiology and Molecular Cell Biology, Eastern Virginia Medical School, Norfolk, VA, USA; ${ }^{3}$ Department of Biostatistics and Health Sciences Research, Mayo Clinic, Rochester, MN, USA; ${ }^{4}$ Department of Laboratory Medicine and Pathology, Mayo Clinic, Rochester, MN, USA; ${ }^{5}$ Department of Epidemiology and Health Sciences Research, Mayo Clinic, Rochester, MN, USA; ${ }^{6}$ Department of Medical Oncology, Mayo Clinic, Rochester, MN, USA; ${ }^{7}$ Department of Radiation Oncology, Mayo Clinic, Phoenix, AZ, USA; ${ }^{8}$ Department of Hepatobiliary \& Pancreas Surgery, Mayo Clinic, Rochester, MN, USA

Contributions: (I) Conception and design: AH Tang, GM Petersen, KW Merrell; (II) Administrative support: GM Petersen; (III) Provision of study materials or patients: GM Petersen, A Mahipal, JB Ashman, WG Rule, D Owen, MA Neben Wittich, RR McWilliams, T Halfdanarson, WW Ma, TT Sio, SP Cleary, MJ Truty, MG Haddock, CL Hallemeier, KW Merrell; (IV) Collection and assembly of data: HC Gits, AH Tang, RP Graham, TC Smyrk, KW Merrell; (V) Data analysis and interpretation: HC Gits, AH Tang, WS Harmsen, WR Bamlet, RO Kowalchuk, TC Smyrk, KW Merrell; (VI) Manuscript writing: All authors; (VII) Final approval of manuscript: All authors.

Correspondence to: Kenneth W. Merrell, MD, MS. 200 First Street SW, Rochester, MN 55905, USA. Email: Merrell.Kenneth@mayo.edu.

Background: Previous reports suggest that intact SMAD4 expression is associated with a locally aggressive pancreas cancer phenotype. The objectives of this work were to determine the frequency of intact SMAD4 and its association with patterns of recurrence in patients with upfront resected pancreas cancer receiving adjuvant therapy.

Methods: A tissue microarray was constructed using resected specimens from patients who underwent upfront surgery and adjuvant gemcitabine with no neoadjuvant treatment for pancreas cancer. SMAD4 expression was determined by immunohistochemical staining. Associations of SMAD4 expression and clinicopathologic parameters with clinical outcomes were evaluated using Cox proportional hazard models.

Results: One hundred twenty-seven patients were included with a median follow up of 5.7 years. Most patients had stage $\geq$ pT3 tumors $(75 \%)$ and pN1 (68\%). All patients received adjuvant gemcitabine, and $79 \%$ of patients received adjuvant chemoradiotherapy. Ten (8\%) patients had intact SMAD4 expression. Grade was the only clinicopathologic parameter statistically associated with SMAD4 expression $(\mathrm{P}=0.05)$. Median overall survival was 2.1 years. On univariate analysis, SMAD4 expression was associated with increased locoregional recurrence (hazard ratio 7.0, $\mathrm{P}<0.01$, 95\% confidence interval: 2.8-18.0) but not distant recurrence $(\mathrm{P}=0.06)$ or overall survival $(\mathrm{P}=0.73)$. On multivariable analysis, SMAD4 expression (hazard ratio 9.6, $\mathrm{P}<0.01$, 95\% confidence interval: $3.7-24.8$ ) and adjuvant chemoradiotherapy (hazard ratio 0.3 , $\mathrm{P}=0.01$, 95\% confidence interval: 0.1-0.8) were associated with higher and lower locoregional recurrence, respectively.

Conclusions: In patients with upfront resected pancreas cancer, SMAD4 expression was associated with an increased risk of locoregional recurrence. Prospective evaluation of the frequency of SMAD4 expression and validation of its predictive utility is warranted.

Keywords: Pancreas cancer; chemoradiotherapy (CRT); mothers against decapentaplegic homolog 4 (SMAD4); tissue microarray (TMA); patterns of recurrence

(C) Journal of Gastrointestinal Oncology. All rights reserved. 
Submitted Feb 03, 2021. Accepted for publication Jun 08, 2021.

doi: 10.21037/jgo-21-55

View this article at: https://dx.doi.org/10.21037/jgo-21-55

\section{Introduction}

With an estimated 57,600 new cases in the United States and the lowest 5 -year survival rate of major cancers, pancreas cancer has become the third leading cause of cancer mortality responsible for an estimated 47,050 deaths in 2020 (1-7). Approximately $20 \%$ of patients with clinically localized tumors are surgical candidates, and R1 resection occurs $20-60 \%$ of the time with survival similar to patients with unresectable tumors (8-10). Locoregional recurrence (LRR) is a known independent predictor of overall survival (OS) and the sole cause of death in up to $25 \%$ of patients $(11,12)$. Despite use of adjuvant chemotherapy and/or chemoradiotherapy (CRT) to mitigate recurrence risk, prospective clinical trials still show high rates of LRR and distant recurrence (DR) of $20-53 \%$ and $46-86 \%$, respectively $(9,13-18)$.

Mothers Against Decapentaplegic homolog 4 (SMAD4), also known as deleted in pancreatic cancer 4 (DPC4), functions in the transforming growth factor beta (TGF- $\beta$ ) pathway to induce growth suppressive effects in normal development and tumorigenesis (19-25). Inactivation or loss of SMAD4 promotes pancreatic tumor growth through the loss of TGF- $\beta /$ SMAD4-dependent cell cycle arrest and apoptosis, and mutations in SMAD4 are found in the majority of pancreatic adenocarcinomas $(20,26)$. SMAD4 immunohistochemical (IHC) staining can be used to assess tumor SMAD4 expression, which is concordant with gene status in pancreas cancer $(27,28)$.

As a biomarker, SMAD4 expression may predict individual LRR or DR risk to stratify patients into selective treatment paradigms, ultimately with the potential to improve survival $(11,27,29-38)$. Past reports suggest loss of SMAD4 expression is associated with a distant metastatic predominant phenotype $(11,28,30)$ and worse prognosis (27,31-38), while intact SMAD4 expression is associated with a locally aggressive tumor phenotype $(11,29)$. For example, in a study of 65 pancreas cancer specimens analyzed on rapid autopsy, $78 \%$ of patients without metastases had intact SMAD4 expression compared to 33\% of patients with metastatic disease (11). In another study, $11(73 \%)$ of 15 patients with intact SMAD4 expression had a locally aggressive pattern of progression compared to four (29\%) of 14 patients with SMAD4 loss (29). Previous studies reveal a range of SMAD4 loss in $15-82 \%$ of resected pancreas tumors $(23,27,30-38)$. The wide range of frequency of SMAD4 loss may reflect differences in patient selection (localized $v s$. metastatic), specimen size, tumor heterogeneity, and IHC staining technique (30,31,36-38).

This work aims to determine the frequency of intact SMAD4 expression in a single-institution cohort of patients with treatment naïve, resected pancreas cancer and to evaluate the association of SMAD4 status with patterns of recurrence and survival. The following article is presented in accordance with the REMARK reporting checklist (39) (available at http://dx.doi.org/10.21037/jgo-21-55).

\section{Methods}

\section{Patient selection}

The study was conducted in accordance with the Declaration of Helsinki (as revised in 2013). The study was approved by the institutional review board of Mayo Clinic (No.: 17-003122), and informed consent was taken from all the patients. From 2000 to 2010, 778 patients with clinically non-metastatic pancreas cancer who underwent curative intent surgery were screened for enrollment in a prospective single-institution clinical registry (Biospecimen Resource for Pancreas Research, Mayo Clinic IRB\#354-06, supported in part by the Mayo Clinic SPORE in Pancreatic Cancer) (40). Of these, 579 patients consented for prospective patient registry enrollment providing access to their medical records and archived tumor tissues. As part of a subsequent retrospective institutional review board-approved study (No.: 17-003122), the pathology biorepository was queried for surgical pathology specimens with sufficient available tissue for tissue microarray (TMA) construction for tumor biomarker analysis. Eligible patients were those who underwent upfront surgery without neoadjuvant therapy and received at least one cycle of postoperative adjuvant gemcitabine.

\section{Tissue microarray generation}

Treatment-naïve tumor biospecimens from 146 patients with formalin-fixed paraffin-embedded tissue available were included in TMA construction. In consideration of 
immense tumor heterogeneity, up to three viable tumor locations were marked by a pathologist from H\&E stained slides. The identified locations were then core punched (2.0 mm diameter) and used to construct a set of TMA blocks (41). Each TMA slide consisted of 60 cores with four cores of non-pancreas tissue samples placed to enable TMA orientation as well as serve as internal controls. IHC staining for SMAD4 was performed using murine B-8 monoclonal Santa Cruz antibody (catalog number SC-7966). TMAs were assessed for staining quality and scored by the study pathologist, who was otherwise blinded to patient clinicopathologic features and clinical outcomes. Scoring based on two measures: percentage of tumor cell staining, ranged $0-100 \%$ in increments of $10 \%$, and staining intensity, scored as $0,1,2$ or 3 (none, weak, moderate, or strong, respectively). A histoscore ranging from 0-300 was created by the product of the percentage of cell staining and tumor intensity. A histoscore greater than zero was considered to have intact SMAD4 expression.

\section{Clinical data collection}

All 127 patients with SMAD4 IHC staining were included in the final analysis. Clinicopathologic features and clinical outcomes of the patients (OS, LRR, and DR) were collected retrospectively from the electronic medical record. LRR was defined by radiographic recurrence within the surgical bed, remnant pancreas, or draining lymph node regions such as superior mesenteric or celiac. DR included recurrences in the lung, liver, and other less common sites. LRR and DR events were tracked independently, and thus patients could be characterized as having one or both types of recurrence, whether identified concurrently or sequentially. The end of the follow up period was 1 January 2020, and median follow-up was 5.7 years (IQR, 4.6-7.6 years).

\section{Statistical analysis}

Descriptive statistics were reported using the number (percentage) for discrete variables and the median and interquartile range (IQR) for continuous variables. The Kaplan Meier method was used to estimate OS. Cumulative incidence estimates were calculated for LRR and DR considering death as a competing risk. Univariate associations of clinicopathologic parameters as variables (SMAD4 expression, resection margins (R0, R1, R2), tumor diameter dichotomized at median, tumor stage dichotomized as T1/T2 versus T3/T4, nodal stage, grade dichotomized at grade 2 versus grade 3/4, lymphovascular invasion, perineural invasion, carbohydrate antigen (CA) 19-9 dichotomized at median, and use of adjuvant CRT) and OS were made using a Cox model. Associations with both LRR and DR were made using the Fine and Gray extension of the Cox model. For the outcomes with significant univariate associations, multivariable models were examined including as candidate variables those with a univariate significance of $\mathrm{P} \leq 0.25$. Variables were retained for the final model using the backward selection method. An alpha level of 0.05 was used for statistical significance.

\section{Results}

Clinicopathologic features of the 127 patients included in the final analysis are summarized in Table 1. All patients received adjuvant gemcitabine with a median number of cycles of 6 (range, 1-6). Seventy-nine percent $(n=99)$ of patients received adjuvant CRT with a median dose fractionation of 5,040 cGy (range, 4,500-5,940 cGy) in 28 fractions (range, 25-33 fractions).

A total of $10(8 \%)$ patients had intact SMAD4 expression and were more likely to have grade 2 tumors $(25 \%$ expression) relative to grade 3 or 4 ( $6 \%$ expression) $(\mathrm{P}=0.05)$. No other clinicopathologic parameters were associated with SMAD4 expression. The median OS for all patients was 2.1 years (IQR, 1.3-3.8 years). Eighty-eight patients experienced any type of recurrence, and of those, 12 patients experienced both LRR and DR. Allowing for recurrences to be assessed independently, LRR and DR events occurred in 20 and 80 patients, respectively (Table 2).

On univariate analysis, intact SMAD4 expression was associated with a higher rate of $\mathrm{LRR}(\mathrm{HR}=7.0, \mathrm{P}<0.01,95 \%$ CI: 2.8-18.0) (Table 3 and Figure 1). SMAD4 expression, use of CRT $(\mathrm{P}=0.07)$, and grade $(\mathrm{P}=0.08)$ were included as candidate variables in the multivariable model for LRR. On multivariable analysis, intact SMAD4 expression was associated with a higher rate of LRR (HR 9.6, P $\leq 0.01$, 95\% CI: 3.7-24.8), use of adjuvant CRT was associated with a lower rate of $\mathrm{LRR}(\mathrm{HR}=0.3, \mathrm{P}=0.01,95 \% \mathrm{CI}: 0.1-0.8)$, and grade was not retained (Table 4). SMAD4 expression was not associated with $\mathrm{DR}(\mathrm{HR}=1.8, \mathrm{P}=0.06,95 \% \mathrm{CI}: 1.0-3.2)$ or $\mathrm{OS}(\mathrm{HR}=1.1, \mathrm{P}=0.73$, 95\% CI: 0.6-2.3) on univariate analyses (Table 3 and Figure 1). As no clinicopathologic parameters were associated significantly with DR or OS on univariate analyses, multivariable analyses were not performed for DR or OS. 
Table 1 Clinicopathologic features of 127 patients included in tissue microarray analysis.

\begin{tabular}{|c|c|c|c|c|}
\hline & All patients, \% [n] $(n=127)$ & SMAD4 intact, \% [n] $(n=10)$ & SMAD4 lost, \% [n] $(n=117)$ & $P$ value \\
\hline Female & $46.5[59]$ & $50.0[5]$ & $46.2[54]$ & \\
\hline Male & $53.5[68]$ & $50.0[5]$ & $53.8[63]$ & \\
\hline Age & & & & 0.61 \\
\hline Interquartile range (years) & $55-73$ & $56-74$ & $55-73$ & \\
\hline ECOG & & & & 0.42 \\
\hline 0 & $84.3[107]$ & $100.0[10]$ & $82.3[97]$ & \\
\hline 1 & $12.6[16]$ & $0.0[0]$ & $13.7[16]$ & \\
\hline Charlson comorbidity index & & & & 0.62 \\
\hline $0-2$ & $19.7[25]$ & $20.0[2]$ & 19.7 [23] & \\
\hline $3-5$ & $56.7[72]$ & $70.0[7]$ & $55.6[65]$ & \\
\hline$>5$ & $21.3[27]$ & $10.0[1]$ & $22.2[26]$ & \\
\hline Missing & $2.4[3]$ & $0.0[0]$ & $2.6[3]$ & \\
\hline Histology & & & & 0.69 \\
\hline Adenocarcinoma & $93.7[119]$ & $100.0[10]$ & $93.2[109]$ & \\
\hline Mucinous carcinoma & $3.9[5]$ & $0.0[0]$ & $4.3[5]$ & \\
\hline Overlap/not specified & $8.7[11]$ & $10.0[1]$ & $8.5[10]$ & \\
\hline Resection margin & & & & 0.72 \\
\hline Ro & 81.9 [104] & $80.0[8]$ & $82.1[96]$ & \\
\hline $\mathrm{R} 1$ & $16.5[21]$ & $20.0[2]$ & $16.2[19]$ & \\
\hline $\mathrm{R} 2$ & $1.6[2]$ & $0.0[0]$ & $1.7[2]$ & \\
\hline Median tumor size & & & & 0.55 \\
\hline Median (mm) & 35.0 & 30.5 & 35.0 & \\
\hline Interquartile range $(\mathrm{mm})$ & $26.0-45.0$ & $27.0-36.0$ & $26.0-45.0$ & \\
\hline Pathologic T stage & & & & 1.00 \\
\hline $\mathrm{T} 1 / \mathrm{T} 2$ & 25.2 [32] & $20.0[2]$ & $25.6[30]$ & \\
\hline T3/T4 & 74.8 [95] & $80.0[8]$ & $74.4[87]$ & \\
\hline
\end{tabular}

Table 1 (continued) 
Table 1 (continued)

\begin{tabular}{|c|c|c|c|c|}
\hline & All patients, $\%[n](n=127)$ & SMAD4 intact, \% [n] $(n=10)$ & SMAD4 lost, \% [n] $(n=117)$ & $P$ value \\
\hline No & $32.3[41]$ & $20.0[2]$ & $33.3[39]$ & 0.50 \\
\hline $\mathrm{N} 1$ & $67.7[86]$ & $80.0[8]$ & $66.7[78]$ & \\
\hline Grade & & & & 0.05 \\
\hline 3 & 76.4 [97] & $50.0[5]$ & 78.6 [92] & \\
\hline 4 & $14.2[18]$ & $20.0[2]$ & $13.7[16]$ & \\
\hline Lymphovascular invasion & & & & 0.62 \\
\hline Absent & $86.6[110]$ & $80.0[8]$ & $87.2[102]$ & \\
\hline Absent & $31.5[40]$ & $80.0[8]$ & $68.5[87]$ & \\
\hline Present & $68.5[87]$ & $20.0[2]$ & $31.5[40]$ & \\
\hline Preoperative CA 19-9 & & & & 0.17 \\
\hline Median $(\mathrm{U} / \mathrm{mL})$ & 195 & 63 & 219 & \\
\hline Interquartile range $(\mathrm{U} / \mathrm{mL})$ & $49-573$ & $26-601$ & $52-553$ & \\
\hline Adjuvant chemoradiotherapy & & & & 0.69 \\
\hline No & 21.4 [27] & $10.0[1]$ & $22.4[26]$ & \\
\hline Yes & 78.6 [99] & $90.0[9]$ & $77.6[90]$ & \\
\hline
\end{tabular}

Table 2 Cumulative incidence and patterns of recurrence by SMAD4 expression

\begin{tabular}{lccc}
\hline & Total events $(\mathrm{n}=127)$ & $\begin{array}{c}\text { 3-year cumulative incidence, } \\
\text { SMAD4 intact (95\% Cl), \% }\end{array}$ & $\begin{array}{c}\text { 3-year cumulative incidence, } \\
\text { SMAD4 lost (95\% Cl), \% }\end{array}$ \\
\hline Any recurrence & 88 & $90.0(73.2-100.0)$ & $61.9(53.6-71.4)$ \\
Locoregional recurrence & 20 & $60.0(36.2-99.5)$ & $10.3(6.1-17.7)$ \\
Distant recurrence & 80 & $80.0(58.7-100.0)$ & $56.7(48.3-66.5)$ \\
\hline
\end{tabular}

$\mathrm{Cl}$, confidence interval.

\section{Discussion}

Pancreas cancer exhibits immense tumor and phenotypic heterogeneity with different treatment responses and patterns of recurrence (42-48). This is likely due to heterogeneous biologic subtypes as a result rapid clonal evolution and selection response $(49,50)$. To date, limited information is available for identification and prognostic evaluation of subtypes for risk stratification. In the postoperative setting, adverse pathologic features and CA 19-9 are correlated retrospectively with LRR and DR (12,51). Unfortunately, these indicators alone are inadequate to predict patterns of recurrence prospectively for guidance of appropriate adjuvant therapies tailored specifically to individual patients. Further, as recent clinical trials provide supportive evidence for neoadjuvant therapy (52-54), 
Table 3 Univariate analyses for locoregional recurrence, distant recurrence, and overall survival

\begin{tabular}{|c|c|c|c|c|c|c|c|c|c|}
\hline & \multicolumn{3}{|c|}{$\begin{array}{l}\text { Univariate analysis for } \\
\text { locoregional recurrence }\end{array}$} & \multicolumn{3}{|c|}{$\begin{array}{l}\text { Univariate analysis for } \\
\text { distant recurrence }\end{array}$} & \multicolumn{3}{|c|}{$\begin{array}{l}\text { Univariate analysis for } \\
\text { overall survival }\end{array}$} \\
\hline & $P$ value & $\mathrm{HR}$ & $95 \% \mathrm{Cl}$ & $P$ value & $\mathrm{HR}$ & $95 \% \mathrm{Cl}$ & $P$ value & $\mathrm{HR}$ & $95 \% \mathrm{Cl}$ \\
\hline \multicolumn{10}{|c|}{ SMAD4 expression } \\
\hline Lost & & 1.00 & & & 1.00 & & & 1.00 & \\
\hline Ro & & 1.00 & & & 1.00 & & & 1.00 & \\
\hline $\mathrm{R} 1$ & 0.53 & 0.62 & $0.15-2.69$ & 0.91 & 1.04 & $0.56-1.92$ & 0.12 & 1.48 & $0.90-2.44$ \\
\hline $\mathrm{R} 2$ & 0.43 & 0.42 & $0.17-65.05$ & 0.68 & 0.55 & $0.03-9.21$ & 0.06 & 3.82 & $0.93-15.72$ \\
\hline \multicolumn{10}{|l|}{ T stage } \\
\hline $1 / 2$ & & 1.00 & & & 1.00 & & & 1.00 & \\
\hline $3 / 4$ & 0.80 & 1.14 & $0.41-3.16$ & 0.96 & 1.01 & $0.62-1.67$ & 0.27 & 1.30 & $0.82-2.05$ \\
\hline \multicolumn{10}{|l|}{$\mathrm{N}$ stage } \\
\hline NO & & 1.00 & & & 1.00 & & & 1.00 & \\
\hline $\mathrm{N} 1$ & 0.54 & 1.37 & $0.50-3.78$ & 0.77 & 0.93 & $0.58-1.49$ & 0.52 & 0.87 & $0.58-1.32$ \\
\hline \multicolumn{10}{|l|}{ Grade } \\
\hline \multicolumn{10}{|c|}{ Perineural invasion } \\
\hline No & & 1.00 & & & 1.00 & & & 1.00 & \\
\hline Yes & 0.30 & 0.56 & $0.19-1.67$ & 0.56 & 1.16 & $0.71-1.87$ & 0.42 & 1.19 & $0.78-1.81$ \\
\hline \multicolumn{10}{|c|}{ Preoperative CA 19-9 } \\
\hline$\leq 195$ & & 1.00 & & & 1.00 & & & 1.00 & \\
\hline$>195$ & 0.89 & 1.07 & $0.44-2.64$ & 0.34 & 1.25 & $0.79-1.95$ & 0.28 & 1.25 & $0.83-1.86$ \\
\hline \multicolumn{10}{|c|}{ Adjuvant chemoradiotherapy } \\
\hline No & & 1.00 & & & 1.00 & & & 1.00 & \\
\hline Yes & 0.07 & 0.43 & $0.17-1.08$ & 0.75 & 0.92 & $0.54-1.56$ & 0.48 & 0.85 & $0.53-1.35$ \\
\hline
\end{tabular}

$\mathrm{HR}$, hazard ratio; $\mathrm{Cl}$, confidence interval. 

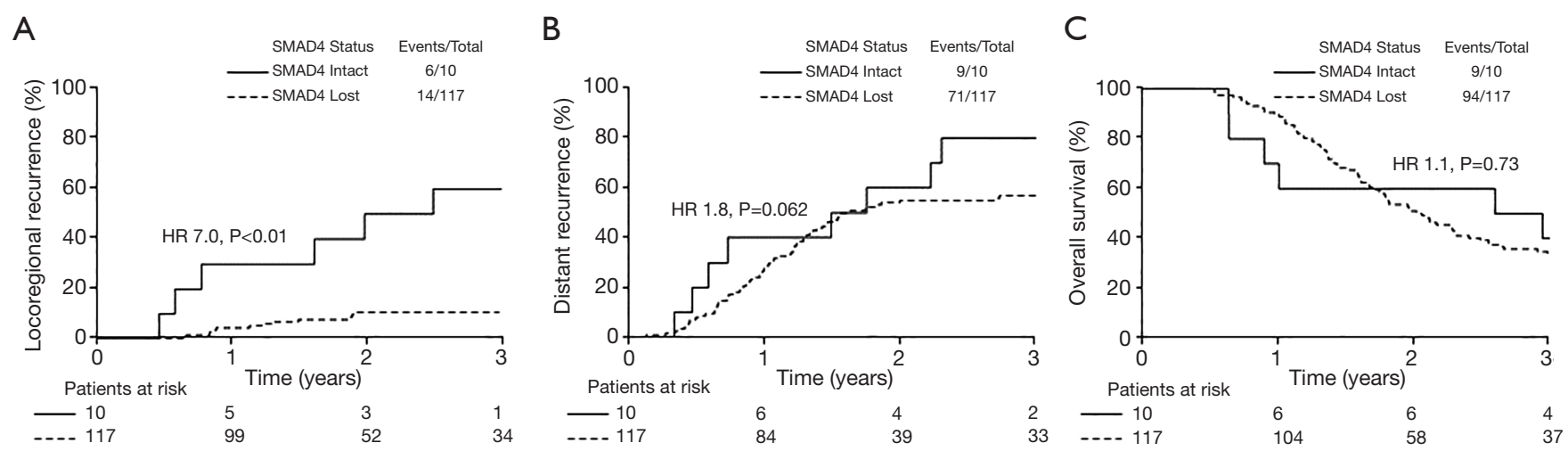

Figure 1 Locoregional recurrence, distant recurrence, and overall survival by SMAD4 immunohistochemical expression: (A) Cumulative incidence of locoregional recurrence; (B) Cumulative incidence of distant recurrence; (C) Kaplan-Meier estimate of overall survival. HR, hazard ratio; CI, confidence interval.

Table 4 Multivariable analysis for locoregional recurrence

\begin{tabular}{lccc}
\hline & & Multivariate analysis for locoregional recurrence & 95\% Cl \\
\cline { 2 - 3 } SMAD4 expression & P value & HR & \\
Lost & & 1.00 & $3.72-24.79$ \\
Intact & $<0.01$ & 9.60 & \\
Adjuvant chemoradiotherapy & & 1.00 \\
No & & 0.31 \\
Yes & 0.01 & $0.12-0.78$ \\
\hline
\end{tabular}

Grade was not retained in the parsimonious model including variables, $\mathrm{P}<0.05$. HR, hazard ratio; $\mathrm{Cl}$, confidence interval.

historical pathologic predictors used to guide post-operative therapy are not available for use in selection of neoadjuvant therapy. Therefore, understanding tumor biology and easily assessable predictive biomarkers would be valuable in order to move toward individualized cancer therapy for pancreas cancer.

The present study adds to the existing body of work by suggesting intact SMAD4 as an independent risk factor for LRR in pancreas cancer. In the present study, 127 patients with resected pancreas cancer treated with adjuvant gemcitabine and in the majority of cases adjuvant CRT were evaluated, and SMAD4 was observed to be intact in $8 \%$ of specimens. This subset of patients treated at the study institution was chosen to isolate better the potential association of SMAD4 status and patterns of recurrence within a historical standard of care. The 3-year cumulative incidences of LRR were $60 \%$ in patients with SMAD4 intact and $10 \%$ in patients with SMAD4 lost. Intact
SMAD4 was predictive of a seven-fold increased risk of LRR on univariate analysis and a ten-fold increased risk of LRR on multivariable analysis, although SMAD4 expression was not associated significantly with DR. Clinicopathologic features were similar between patients with intact or lost SMAD4 with the exception of histopathologic grade, which was not associated with LRR on univariate or multivariable analysis. These data support the hypothesis that intact SMAD4 expression may be an independent predictor of a pancreas cancer subset with a predilection for LRR and may be used in risk stratification to identify patients who may be most likely to benefit from aggressive locoregional therapy.

Recent clinical trials provide evidence for shifting the standard treatment paradigm of non-metastatic pancreas cancer to upfront neoadjuvant chemotherapy and CRT prior to an attempt at surgical resection (52-57). Two randomized trials demonstrated the benefits of neoadjuvant CRT prior to resection compared to upfront resection 
with adjuvant chemotherapy or CRT $(52,54,58)$. While neoadjuvant CRT led to improvements in locoregional failure and disease-free interval, an additional benefit was the ability to detect the manifestation of occult metastatic disease during and after CRT but before surgery, thus improving the selection of surgical cases $(54,58)$. For example, in the PREOPANC clinical trial, approximately $14 \%$ of the patients who were randomized to and received neoadjuvant CRT did not undergo resection due to manifestation of metastatic disease $(54,58)$. In a predefined subgroup analysis, patients who underwent tumor resection and started adjuvant therapy had a median OS of 35.2 vs. 19.8 months in the immediate surgery group. Despite these advantages, the rates of distant metastasis were similar between groups. These findings indicate that current clinical tools are inadequate to determine individual biology and to predict patterns of progression. Thus, a major challenge in treatment of pancreas cancer, with or without a neoadjuvant approach, is selecting appropriate patients for whom aggressive locoregional therapy may prolong survival.

Most studies have evaluated SMAD4 expression in the context of treatment-naïve, resected pancreas cancer. It is uncertain as to what impact SMAD4 expression may have in patients who undergo neoadjuvant treatment. In one study, the frequency of SMAD4 expression of resected specimens was similar between patients who did and did not receive neoadjuvant treatment ( $20 \%$ vs. $13 \%$, respectively), and SMAD4 loss was associated with a shorter time to DR in the combined cohort $(\mathrm{P}=0.02)$ (30). As SMAD4 staining may be performed on fine needle aspiration samples with accuracy $(34,59,60)$, it is feasible to assess SMAD4 status at diagnosis prior to neoadjuvant treatment as well as at possible resection after neoadjuvant treatment. It remains unclear whether neoadjuvant chemotherapy or CRT may change the expression of, or patterns of recurrence predicted by, SMAD4 and thus its relevance in a neoadjuvant treatment paradigm.

Beyond SMAD4, other molecular markers of loss of tumor suppression or oncogene activation have been explored for predictive utility of patterns of recurrence. Examples of significant correlations include inactivating mutations in TP53 with a distant metastatic predominant phenotype (11), high CXCR4 expression with a DR pattern (61), loss of p16 with DR as first recurrence and dominant pattern of progression (34), and high c-MET with shortened time to DR (30). While each of these markers are of interest, a synergistic combination potentially could be used to enhance their clinical utility (41). A panel of predictive and prognostic biomarkers may provide a unique signature that could be used to guide the treatment sequences of different combinatorial therapies to improve survival among various pancreas cancer subtypes.

While the results of this study are hypothesis generating and encouraging, several limitations are present. First, this is a selected cohort of patients from a single medical institution, and all inherent biases of a retrospective study may be present. The frequency of intact SMAD4 expression $(8 \%)$ was lower than those of previously published studies, which may reflect an institution-based selection effect of treating patients with more advanced pancreas cancer and may limit the generalizability of the findings (30,31,36-38). The low detected frequency of intact SMAD4 also could reflect sampling error caused by tumor heterogeneity and the relatively small samples utilized for TMA construction (41). To attempt to mitigate this, up to three separate cores were taken per tumor. Further, this study was performed without a validation cohort, and validation of these results with an independent cohort is crucial. While lacking a true validation cohort, the observations of this study fit within the context of previously published literature and suggest a relationship between intact SMAD4 expression and LRR. Ultimately the findings are correlational and prospective evaluation is warranted.

In conclusion, in a cohort of patients with resected pancreas cancer, intact SMAD4 expression was associated with a locally aggressive phenotype with a markedly increased risk of LRR. Multivariable analysis demonstrated intact SMAD4 and no adjuvant CRT were associated with increased risk of LRR. This work contributes to a growing body of knowledge that attempts to identify and differentiate molecular signatures in pancreas cancer and stratify patients who are more likely to benefit from aggressive locoregional therapy, including CRT. Future directions include prospective evaluation of the frequency of intact SMAD4, validation of its predictive utility including in both the adjuvant and neoadjuvant settings, and investigation into other biomarkers that could be combined with SMAD4 for improved prognostication.

\section{Acknowledgments}

We thank the pancreas cancer patients for contributing to this project at the Mayo Clinic as well as our colleagues in the Department of Radiation Oncology and the Mayo Clinic Pancreatic Cancer SPORE for their expert guidance 
and generous support.

Funding: This work was supported by National Cancer Institute (Mayo Clinic SPORE in Pancreatic Cancer P50CA102701 to GMP, CA140550 to AHT); 2010 AACRPanCAN Innovative Grant (AACR-PanCan \#169458 to AHT); 2005 Lutsgarten Foundation for Pancreatic Cancer Research (RFA05-046 to AHT); National Institute of General Medical Sciences (GM069922-06S1 to AHT); Dorothy G. Hoefer Foundation (to AHT); and the Mayo Pancreatic Cancer SPORE Pilot Grant Award (to AHT).

\section{Footnote}

Reporting Checklist: The authors have completed the REMARK reporting checklist available at http://dx.doi. org/10.21037/jgo-21-55

Data Sharing Statement: Available at http://dx.doi. org/10.21037/jgo-21-55

Peer Review File: Available at http://dx.doi.org/10.21037/ jgo-21-55

Conflicts of Interest: All authors have completed the ICMJE uniform disclosure form (available at http://dx.doi. org/10.21037/jgo-21-55). SPC declares Olympus consulting and Ethicon educational events. ROK declares his wife is a senior technical product manager for GE Healthcare. KWM declares ongoing grant support for clinical trial and global medical education programs. None of these grants are relevant to the manuscript. DO declares grants for clinical trial operations and protocol delivery from Mayo Clinic and Astra Zeneca. No direct financial relationship. TTS declares that he provides strategic and scientific recommendations as a member of the Advisory Board and speaker for Novocure, Inc., which is not in any way associated with the content or disease site as presented in this manuscript. AHT declares grants from AACR-PanCAN, Lutsgarten Foundation for Pancreatic Cancer Research, National Institute of General Medical Sciences, National Cancer Institute, and Dorothy G. Hoefer Foundation for Pancreatic Cancer Research, is a grant reviewer at National Institute of Health, National Cancer Institute, Department of Defense, and PanCAN, and is a Vice Chair at PanCAN Career Development Award Scientific Review Committee. The other authors have no conflicts of interest to declare.

Ethical Statement: The authors are accountable for all aspects of the work in ensuring that questions related to the accuracy or integrity of any part of the work are appropriately investigated and resolved. The study was conducted in accordance with the Declaration of Helsinki (as revised in 2013). The study was approved by the institutional review board of Mayo Clinic (No.: 17-003122), and informed consent was taken from all the patients. All figures and tables in this manuscript are original and are not adapted from published ones.

Open Access Statement: This is an Open Access article distributed in accordance with the Creative Commons Attribution-NonCommercial-NoDerivs 4.0 International License (CC BY-NC-ND 4.0), which permits the noncommercial replication and distribution of the article with the strict proviso that no changes or edits are made and the original work is properly cited (including links to both the formal publication through the relevant DOI and the license). See: https://creativecommons.org/licenses/by-nc-nd/4.0/.

\section{References}

1. Siegel RL, Miller KD, Jemal A. Cancer statistics, 2020. CA Cancer J Clin 2020;70:7-30.

2. Hruban RH, Gaida MM, Thompson E, et al. Why is pancreatic cancer so deadly? The pathologist's view. J Pathol 2019;248:131-41.

3. Garrido-Laguna I, Hidalgo M. Pancreatic cancer: from state-of-the-art treatments to promising novel therapies. Nat Rev Clin Oncol 2015;12:319-34.

4. Kleeff J, Korc M, Apte M, et al. Pancreatic cancer. Nat Rev Dis Primers 2016;2:16022.

5. Rahib L, Smith BD, Aizenberg R, et al. Projecting cancer incidence and deaths to 2030: the unexpected burden of thyroid, liver, and pancreas cancers in the United States. Cancer Res 2014;74:2913-21.

6. Sakamoto H, Attiyeh MA, Gerold JM, et al. The Evolutionary Origins of Recurrent Pancreatic Cancer. Cancer Discov 2020;10:792-805.

7. Wolfgang CL, Herman JM, Laheru DA, et al. Recent progress in pancreatic cancer. CA Cancer J Clin 2013;63:318-48.

8. Howard TJ, Krug JE, Yu J, et al. A margin-negative R0 resection accomplished with minimal postoperative complications is the surgeon's contribution to longterm survival in pancreatic cancer. J Gastrointest Surg 2006;10:1338-45; discussion 1345-6.

9. Neoptolemos JP, Palmer DH, Ghaneh P, et al. Comparison 
of adjuvant gemcitabine and capecitabine with gemcitabine monotherapy in patients with resected pancreatic cancer (ESPAC-4): a multicentre, open-label, randomised, phase 3 trial. Lancet 2017;389:1011-24.

10. Raut CP, Tseng JF, Sun CC, et al. Impact of resection status on pattern of failure and survival after pancreaticoduodenectomy for pancreatic adenocarcinoma. Ann Surg 2007;246:52-60.

11. Iacobuzio-Donahue CA, Fu B, Yachida S, et al. DPC4 gene status of the primary carcinoma correlates with patterns of failure in patients with pancreatic cancer. J Clin Oncol 2009;27:1806-13.

12. Merrell KW, Haddock MG, Quevedo JF, et al. Predictors of Locoregional Failure and Impact on Overall Survival in Patients With Resected Exocrine Pancreatic Cancer. Int J Radiat Oncol Biol Phys 2016;94:561-70.

13. Kalser MH, Ellenberg SS. Pancreatic cancer. Adjuvant combined radiation and chemotherapy following curative resection. Arch Surg 1985;120:899-903.

14. Neoptolemos JP, Dunn JA, Stocken DD, et al. Adjuvant chemoradiotherapy and chemotherapy in resectable pancreatic cancer: a randomised controlled trial. Lancet 2001;358:1576-85.

15. Neoptolemos JP, Stocken DD, Friess H, et al. A randomized trial of chemoradiotherapy and chemotherapy after resection of pancreatic cancer. N Engl J Med 2004;350:1200-10.

16. Regine WF, Winter KA, Abrams RA, et al. Fluorouracil vs gemcitabine chemotherapy before and after fluorouracilbased chemoradiation following resection of pancreatic adenocarcinoma: a randomized controlled trial. JAMA 2008;299:1019-26.

17. Smeenk HG, van Eijck CH, Hop WC, et al. Longterm survival and metastatic pattern of pancreatic and periampullary cancer after adjuvant chemoradiation or observation: long-term results of EORTC trial 40891. Ann Surg 2007;246:734-40.

18. Conroy T, Hammel P, Hebbar M, et al. FOLFIRINOX or Gemcitabine as Adjuvant Therapy for Pancreatic Cancer. N Engl J Med 2018;379:2395-406.

19. Derynck R, Zhang YE. Smad-dependent and Smadindependent pathways in TGF-beta family signalling. Nature 2003;425:577-84.

20. Ahmed S, Bradshaw AD, Gera S, et al. The TGF- $\beta / \operatorname{Smad} 4$ Signaling Pathway in Pancreatic Carcinogenesis and Its Clinical Significance. J Clin Med 2017;6:5.

21. Huang W, Navarro-Serer B, Jeong YJ, et al. Pattern of Invasion in Human Pancreatic Cancer Organoids Is
Associated with Loss of SMAD4 and Clinical Outcome. Cancer Res 2020;80:2804-17.

22. Pokataev I, Kudaibergenova A, Artemyeva A, et al. Intratumoral Heterogeneity of SMAD4 Immunohistochemical Expression and Its Role in Prediction of Recurrence Pattern in Patients with Resectable Pancreatic Cancer. J Gastrointest Cancer 2019;50:478-84.

23. Shugang X, Hongfa Y, Jianpeng L, et al. Prognostic Value of SMAD4 in Pancreatic Cancer: A Meta-Analysis. Transl Oncol 2016;9:1-7.

24. Wang F, Xia X, Yang C, et al. SMAD4 Gene Mutation Renders Pancreatic Cancer Resistance to Radiotherapy through Promotion of Autophagy. Clin Cancer Res 2018;24:3176-85.

25. Yokose T, Kitago M, Matsuda S, et al. Combination of KRAS and SMAD4 mutations in formalin-fixed paraffinembedded tissues as a biomarker for pancreatic cancer. Cancer Sci 2020;111:2174-82.

26. Furukawa T, Sunamura M, Horii A. Molecular mechanisms of pancreatic carcinogenesis. Cancer Sci 2006;97:1-7.

27. Tascilar M, Skinner HG, Rosty C, et al. The SMAD4 protein and prognosis of pancreatic ductal adenocarcinoma. Clin Cancer Res 2001;7:4115-21.

28. Yachida S, White CM, Naito Y, et al. Clinical significance of the genetic landscape of pancreatic cancer and implications for identification of potential long-term survivors. Clin Cancer Res 2012;18:6339-47.

29. Crane CH, Varadhachary GR, Yordy JS, et al. Phase II trial of cetuximab, gemcitabine, and oxaliplatin followed by chemoradiation with cetuximab for locally advanced (T4) pancreatic adenocarcinoma: correlation of Smad4(Dpc4) immunostaining with pattern of disease progression. J Clin Oncol 2011;29:3037-43.

30. Cuneo KC, Morgan MA, Griffith KA, et al. Prognostic Value of c-MET Expression in Patients With Pancreatic Cancer Receiving Adjuvant and Neoadjuvant Chemoradiation Therapy. Int J Radiat Oncol Biol Phys 2018;100:490-7.

31. Hua Z, Zhang YC, Hu XM, et al. Loss of DPC4 expression and its correlation with clinicopathological parameters in pancreatic carcinoma. World J Gastroenterol 2003;9:2764-7.

32. Jiang H, He C, Geng S, et al. RhoT1 and Smad4 are correlated with lymph node metastasis and overall survival in pancreatic cancer. PLoS One 2012;7:e42234.

33. Kadera BE, Sunjaya DB, Isacoff WH, et al. Locally 
advanced pancreatic cancer: association between prolonged preoperative treatment and lymph-node negativity and overall survival. JAMA Surg 2014;149:145-53.

34. Oshima M, Okano K, Muraki S, et al. Immunohistochemically detected expression of 3 major genes (CDKN2A/p16, TP53, and SMAD4/DPC4) strongly predicts survival in patients with resectable pancreatic cancer. Ann Surg 2013;258:336-46.

35. Ottenhof NA, Morsink FH, Ten Kate F, et al. Multivariate analysis of immunohistochemical evaluation of protein expression in pancreatic ductal adenocarcinoma reveals prognostic significance for persistent Smad4 expression only. Cell Oncol (Dordr) 2012;35:119-26.

36. Shin SH, Kim SC, Hong SM, et al. Genetic alterations of K-ras, p53, c-erbB-2, and DPC4 in pancreatic ductal adenocarcinoma and their correlation with patient survival. Pancreas 2013;42:216-22.

37. Toga T, Nio Y, Hashimoto K, et al. The dissociated expression of protein and messenger RNA of DPC4 in human invasive ductal carcinoma of the pancreas and their implication for patient outcome. Anticancer Res 2004;24:1173-8.

38. Yamada S, Fujii T, Shimoyama Y, et al. SMAD4 expression predicts local spread and treatment failure in resected pancreatic cancer. Pancreas 2015;44:660-4.

39. McShane LM, Altman DG, Sauerbrei W, et al. Reporting recommendations for tumor marker prognostic studies (REMARK). J Natl Cancer Inst 2005;97:1180-4.

40. Hu C, Hart SN, Polley EC, et al. Association Between Inherited Germline Mutations in Cancer Predisposition Genes and Risk of Pancreatic Cancer. JAMA 2018;319:2401-9.

41. Qin R, Smyrk TC, Reed NR, et al. Combining clinicopathological predictors and molecular biomarkers in the oncogenic K-RAS/Ki67/HIF-1 $\alpha$ pathway to predict survival in resectable pancreatic cancer. Br J Cancer 2015;112:514-22.

42. Cros J, Raffenne J, Couvelard A, et al. Tumor Heterogeneity in Pancreatic Adenocarcinoma. Pathobiology 2018;85:64-71.

43. Roberts NJ, Norris AL, Petersen GM, et al. Whole Genome Sequencing Defines the Genetic Heterogeneity of Familial Pancreatic Cancer. Cancer Discov 2016;6:166-75.

44. Juiz NA, Iovanna J, Dusetti N. Pancreatic Cancer Heterogeneity Can Be Explained Beyond the Genome. Front Oncol 2019;9:246.

45. Le Large TY, Mantini G, Meijer LL, et al. Microdissected pancreatic cancer proteomes reveal tumor heterogeneity and therapeutic targets. JCI Insight 2020;5:138290.

46. Makohon-Moore AP, Zhang M, Reiter JG, et al. Limited heterogeneity of known driver gene mutations among the metastases of individual patients with pancreatic cancer. Nat Genet 2017;49:358-66.

47. Mena E, Sheikhbahaei S, Taghipour M, et al. 18F-FDG PET/CT Metabolic Tumor Volume and Intratumoral Heterogeneity in Pancreatic Adenocarcinomas: Impact of Dual-Time Point and Segmentation Methods. Clin Nucl Med 2017;42:e16-21.

48. Yoon S, Li H, Quintanar L, et al. Uncovering Differently Expressed Markers and Heterogeneity on Human Pancreatic Cancer. Transl Oncol 2020;13:100749.

49. Campbell PJ, Yachida S, Mudie LJ, et al. The patterns and dynamics of genomic instability in metastatic pancreatic cancer. Nature 2010;467:1109-13.

50. Yamamoto KN, Liu LL, Nakamura A, et al. Stochastic Evolution of Pancreatic Cancer Metastases During Logistic Clonal Expansion. JCO Clin Cancer Inform 2019;3:1-11.

51. Regine WF, Winter KA, Abrams R, et al. Fluorouracilbased chemoradiation with either gemcitabine or fluorouracil chemotherapy after resection of pancreatic adenocarcinoma: 5-year analysis of the U.S. Intergroup/ RTOG 9704 phase III trial. Ann Surg Oncol 2011;18:1319-26.

52. Jang JY, Han Y, Lee H, et al. Oncological Benefits of Neoadjuvant Chemoradiation With Gemcitabine Versus Upfront Surgery in Patients With Borderline Resectable Pancreatic Cancer: A Prospective, Randomized, Open-label, Multicenter Phase 2/3 Trial. Ann Surg 2018;268:215-22.

53. Katz MHG, Ou FS, Herman JM, et al. Alliance for clinical trials in oncology (ALLIANCE) trial A021501: preoperative extended chemotherapy vs. chemotherapy plus hypofractionated radiation therapy for borderline resectable adenocarcinoma of the head of the pancreas. BMC Cancer 2017;17:505.

54. Versteijne E, van Eijck CH, Punt CJ, et al. Preoperative radiochemotherapy versus immediate surgery for resectable and borderline resectable pancreatic cancer (PREOPANC trial): study protocol for a multicentre randomized controlled trial. Trials 2016;17:127.

55. Evans DB, Varadhachary GR, Crane CH, et al. Preoperative gemcitabine-based chemoradiation for patients with resectable adenocarcinoma of the pancreatic head. J Clin Oncol 2008;26:3496-502. 
56. Katz MH, Shi Q, Ahmad SA, et al. Preoperative Modified FOLFIRINOX Treatment Followed by CapecitabineBased Chemoradiation for Borderline Resectable Pancreatic Cancer: Alliance for Clinical Trials in Oncology Trial A021101. JAMA Surg 2016;151:e161137.

57. Truty MJ, Kendrick ML, Nagorney DM, et al. Factors Predicting Response, Perioperative Outcomes, and Survival Following Total Neoadjuvant Therapy for Borderline/Locally Advanced Pancreatic Cancer. Ann Surg 2021;273:341-9.

58. Versteijne E, Suker M, Groothuis K, et al. Preoperative Chemoradiotherapy Versus Immediate Surgery for Resectable and Borderline Resectable Pancreatic Cancer: Results of the Dutch Randomized Phase III PREOPANC

Cite this article as: Gits HC, Tang AH, Harmsen WS, Bamlet WR, Graham RP, Petersen GM, Smyrk TC, Mahipal A, Kowalchuk RO, Ashman JB, Rule WG, Owen D, Neben Wittich MA, McWilliams RR, Halfdanarson T, Ma WW, Sio TT, Cleary SP, Truty MJ, Haddock MG, Hallemeier CL, Merrell KW. Intact SMAD-4 is a predictor of increased locoregional recurrence in upfront resected pancreas cancer receiving adjuvant therapy. J Gastrointest Oncol 2021;12(5):22752286. doi: 10.21037/jgo-21-55
Trial. J Clin Oncol 2020;38:1763-73.

59. Ali S, Cohen C, Little JV, et al. The utility of SMAD4 as a diagnostic immunohistochemical marker for pancreatic adenocarcinoma, and its expression in other solid tumors. Diagn Cytopathol 2007;35:644-8.

60. Zapata M, Cohen C, Siddiqui MT. Immunohistochemical expression of SMAD4, CK19, and CA19-9 in fine needle aspiration samples of pancreatic adenocarcinoma: Utility and potential role. Cytojournal 2007;4:13.

61. Bachet JB, Maréchal R, Demetter P, et al. Contribution of CXCR4 and SMAD4 in predicting disease progression pattern and benefit from adjuvant chemotherapy in resected pancreatic adenocarcinoma. Ann Oncol 2012;23:2327-35. 